\title{
Prevalence, predictors and prognosis of incidental intracranial aneurysms in patients with suspected TIA and minor stroke: a population-based study and systematic review
}

\author{
Robert Hurford (1) , Isabel Taveira, Wilhelm Kuker 두, Peter M Rothwell, On behalf of \\ the Oxford Vascular Study Phenotyped Cohort
}

- Additional material is published online only. To view please visit the journal online (http://dx.doi.org/10.1136/ jnnp-2020-324418).

Centre for the Prevention of Stroke and Dementia, University of Oxford, Oxford, UK

\section{Correspondence to} Prof Peter M Rothwell, Centre for the Prevention of Stroke and Dementia, University of Oxford, Oxford, Oxfordshire, UK; peter. rothwell@ndcn.ox.ac.uk

Received 27 June 2020 Revised 17 August 2020 Accepted 7 October 2020 Published Online First 4 November 2020

\section{Check for updates}

(c) Author(s) (or their employer(s)) 2021. Re-use permitted under CC BY. Published by BMJ.

To cite: Hurford R, Taveira I, Kuker W, et al. J Neurol Neurosurg Psychiatry 2021:92:542-548.

\section{ABSTRACT}

Introduction Unruptured intracranial aneurysms (UIAs) are common incidental imaging findings, but there are few data in patients with transient ischaemic attack (TIA)/stroke. The frequency of UIA might be higher due to shared risk factors, but rupture risk might be reduced by intensive secondary prevention. We determined the prevalence and prognosis of UIA in patients with suspected TIA/minor stroke.

Methods All patients referred to the population-based Oxford Vascular Study (2011-2020) with suspected $\mathrm{TIA} /$ minor stroke and non-invasive angiography were included. We determined the prevalence of incidental asymptomatic UIA and the risk of subsequent subarachnoid haemorrhage (SAH) by follow-up on intensive medical treatment, with guideline-based monitoring/management. We also did a systematic review of UIA prevalence/prognosis in cohorts with TIA/ stroke.

Findings Among 2013 eligible patients, 95 (4.7\%) had 103 previously unknown asymptomatic UIA. Female sex (OR 2.3, 95\% Cl 1.5 to 3.7), smoking (2.1, 1.2 to 3.6) and hypertension (1.6, 1.0 to 2.5) were independently predictive of UIA, with a prevalence of $11.1 \%$ in those with all three risk factors. During mean follow-up of 4.5 years, only one SAH occurred: 2.3 (95\% CI 0.3 to 16.6) per 1000 person-years. We identified 19 studies of UIA in TIA/stroke cohorts $(n=12781)$, all with either symptomatic carotid stenosis or major acute stroke. The pooled mean UIA prevalence in patients with TIA/stroke was $5.1 \%(95 \% \mathrm{Cl} 4.8$ to 5.5$)$ and the incidence of $\mathrm{SAH}$ was $4.6(95 \% \mathrm{Cl} 1.9$ to 11.0$)$ per 1000 person-years. Interpretation The $5 \%$ prevalence of UIA in patients with confirmed TIA/minor stroke is likely higher than that in the general population. However, the risk of SAH on intensive medical treatment and guideline-based management/monitoring is low.

\section{INTRODUCTION}

Saccular unruptured intracranial aneurysms (UIAs) are present in $3 \%$ of the adult general population ${ }^{1}$ and are the most common incidental finding on neuroimaging in healthy individuals. ${ }^{2}$ Although the frequency and quality of non-invasive angiography in the assessment of suspected ischaemic stroke or transient ischaemic attack (TIA) is increasing, the optimal management of asymptomatic UIAs remains uncertain. ${ }^{3}$ Moreover, previous studies have been limited to selected cohorts under investigation for symptomatic carotid stenosis or major acute stroke.

Patients referred to acute neurovascular services for suspected TIA or minor stroke also have a greater burden of vascular risk factors and a higher susceptibility to cerebrovascular disease than the general population. ${ }^{4}$ As cerebrovascular disease and intracerebral aneurysm development have risk factors in common, it might be expected that these patients will also have a higher burden of UIAs. However, there have been no population-based studies assessing the prevalence of UIAs in a general cerebrovascular disease cohort and none of patients with TIA and minor stroke.

Furthermore, while there may be a higher prevalence of UIAs in a cerebrovascular disease cohort, it is unknown whether the risk of rupture is comparable with that in the general population. Risk factors for intracerebral aneurysm rupture, such as hypertension and smoking, are common but are more intensively managed after TIA or ischaemic stroke. Moreover, there is some evidence aspirin lowers the risk of intracerebral aneurysm rupture by reducing vessel wall inflammation. ${ }^{5-7}$ As patients with minor stroke and TIA would often be suitable candidates for intervention, the prognosis of incidental UIAs is of clinical importance.

We aimed to determine the prevalence and predictors of UIA in patients referred to an acute neurovascular service with suspected TIA or minor stroke, and to assess the risk of subarachnoid haemorrhage on follow-up with a standard guidelinebased policy for intervention/surveillance. We also did a systematic review and meta-analysis of previous studies to establish the prevalence and prognosis of UIA in patients with TIA or stroke.

\section{METHODS}

The Oxford Vascular Study (OXVASC) is a longitudinal population-based incidence cohort of all acute vascular events in a defined population of 92728 , covered by around 100 primary care physicians in nine primary care practices in Oxfordshire, UK. An estimated $97 \%$ of the true study residential population is registered with a primary care practice; 
most unregistered people are young students. The study area contains a mix of urban and rural populations. The OXVASC population is 94\% Caucasian, 3\% Asian, 2\% Chinese and 1\% Afro-Caribbean. ${ }^{8}$

Detailed methodology of OXVASC has been reported previously. ${ }^{9}$ Multiple overlapping methods were used for ascertainment of all individuals with TIA and stroke, approaching $100 \%$ of events reaching medical attention. These include the following: (1) a daily, rapid access clinic to which participating primary care practitioners and the local emergency department refer individuals with suspected TIA or minor stroke; (2) daily searches of admissions to the medical, stroke, neurology and other relevant wards; (3) daily searches of the local emergency department attendance register; (4) monthly searches of general practitioner diagnostic coding and hospital discharge codes; (5) monthly searches of all brain and vascular imaging referrals. Patients gave written informed consent after an event or assent was obtained from a relative for patients who were unable to provide consent. $^{1011}$

We studied consecutive patients referred to OXVASC rapid access service between 1 March 2011 and 1 March 2020 with suspected TIA or minor ischaemic stroke (National Institute of Health Stroke Scale (NIHSS) score $\leq 3$ ) and consenting to investigation. All patients with intracranial vascular imaging were included in the analyses.

Demographic data and stroke risk factors were collected from face-to-face interview by study physicians as soon as possible after referral or hospital admission and cross-referenced with primary care records. Detailed clinical history was recorded in all patients and assessments were made for stroke severity using the NIHSS as recorded on assessment. Cause of ischaemic events was classified according to the Trial of Org 10172 in Acute Stroke Treatment (TOAST) criteria. $^{11}$ Stroke and TIA were defined according to WHO criteria (acute onset of neurological deficit, persisting for $>24$ hours in case of a stroke or for $<24$ hours in case of a TIA), ${ }^{12}$ with review of all cases as soon as possible after presentation by the same senior neurologist (PMR) throughout the study.

\section{Imaging procedures}

Intracranial vascular imaging was done in all patients in OXVASC from 2011 onward. We aimed to obtain as high an imaging rate as possible by using MR angiography (MRA) as first choice and CT angiography (CTA; Toshiba, Aquilion 64, 64-slice scanner) if MRI was contraindicated (eg, implantable devices or claustrophobia).

The MRI scanners and protocols used in OXVASC have been described elsewhere, ${ }^{13}$ but sequences included diffusionweighted imaging, time of flight angiography of the intracranial arteries and gadolinium contrast-enhanced angiography (CEMRA) of the intracranial and cervicocranial arteries including the aortic arch. Patients were scanned at the Acute Vascular Imaging Centre, John Radcliffe Hospital, in a 3.0 T Siemens Verio scanner; a neurovascular coil was used (CE-MRA sequence: $15 \mathrm{~mL}$ ProHance followed by $40 \mathrm{~mL} \mathrm{NaCl}$, flow rate $2 \mathrm{~mL} / \mathrm{s}$, TR $22 \mathrm{~ms}$, TE $3.6 \mathrm{~ms}$, flip angle $18^{\circ}$, slice thickness $0.5 \mathrm{~mm}$ ).

All imaging was reviewed and reported daily by a single study neuroradiologist (WK) and discussed at a weekly neuroradiology meeting. The number, location and size of UIA was recorded. Saccular aneurysms were defined as an abnormal swelling or bulge of an intracranial artery bifurcation with a distinct neck at the point of attachment to the parent vessel. Non-saccular aneurysms (fusiform aneurysms) were defined as a circumferential wall swelling or distention without a distinct neck; these are reported but not included in the analyses. The 5-year risk of aneurysm rupture was calculated using the PHASES score. ${ }^{14}$ The management of all incidental UIA was determined at a weekly multidisciplinary meeting of interventional neuroradiologists, neurologists and vascular neurosurgeons using a standard policy aligned to the American Heart Association/American Stroke Association guidelines for incidental UIA more generally. ${ }^{15}$

Patients were followed up face to face at 1, 6, 12, 24, 60 and 120 months by a study nurse or physician to identify any recurrent stroke (supplemented by review of primary care records) and to ensure medication compliance and adequate blood pressure control. Patients who had moved out of the study area (or were unwilling/unable to have face-to-face follow-up) were followed up via telephone at the same time points. All recurrent events, including subsequent subarachnoid haemorrhage (SAH), occurring during follow-up would also be identified by the ongoing daily case ascertainment. We recorded all deaths during follow-up with the underlying causes by direct follow-up, via primary care records, and by centralised registration with Office for National Statistics (ONS).

\section{Statistical analysis}

Analyses included all eligible patients with intracranial vascular imaging. Baseline characteristics were compared between patients with and without UIA, subcategorised into discharge diagnosis, using $\chi^{2}$ or Student's t-test as appropriate.

We calculated the age-specific prevalence of UIA in 10-year bands, stratified by sex. We determined the predictors of UIA with univariate and multivariate regression analyses.

We determined the absolute risk of subsequent SAH during follow-up (to 31 March 2020) after the index event and compared this with the predicted risk as calculated by the mean cohort PHASES score.

All statistical analyses were performed with IBM SPSS V.25.0.

\section{Systematic review}

All studies reporting the prevalence of asymptomatic, saccular UIAs in patients with ischaemic stroke or TIA were included. Embase and Ovid MEDLINE databases were searched using the search terms (online supplemental figure 1) from inception until 1 May 2020. Intracranial vascular imaging by any modality was considered. Studies of patients with asymptomatic carotid stenosis only and case reports/series were excluded. The outcome of interest was the proportion of patients with UIAs and the outcome of the UIA. Disagreement over eligibility was resolved by discussion with the senior author (PMR).

\section{Data abstraction and quality assessment}

Key descriptive and quantitative data were recorded for study design, cohort characteristics and outcome data. Recorded details included the year of study publication, study location number of participants, proportion of females, mean cohort age, imaging modality, the prevalence of UIAs and UIA outcome if reported.

\section{Meta-Analysis}

The leading outcome of interest was the prevalence of UIA in the study population. Reported p values were two sided, with significance set $<0.05$. Heterogeneity among included studies was assessed by $\chi^{2}$ statistics. For analysis, we used Review Manager (RevMan), V.5.3; Copenhagen: The Nordic Cochrane Centre, The Cochrane Collaboration, 2014. 
Standard protocol approvals, registrations and patient consents

Written informed consent or assent from relatives was obtained in all participants for study interview and follow-up, including ongoing review of primary care and hospital records and death certificate data. OXVASC was approved by the Oxfordshire research ethics committee (OREC A: 05/Q1604/70).

\section{RESULTS}

Of 2498 eligible patients referred (1143/45.8\% TIA, 695/27.8\% minor ischaemic stroke and 660/26.4\% stroke mimics), 2013 underwent intracranial vascular imaging (1658/82.4\% MRA and $355 / 17.6 \%$ CTA). Patients who did not receive intracranial vascular imaging (principally due to non-vascular diagnosis, dementia or patient refusal) were more likely to be female; have a background of atrial fibrillation, peripheral vascular or ischaemic heart disease and have a non-cerebrovascular discharge diagnosis (online supplemental table 1).

Of the 2013 patients with intracranial vascular imaging, 103 previously unknown, saccular, asymptomatic UIA were identified in 95 (4.7\%) patients. Four non-saccular (fusiform) aneurysms and one previously coiled aneurysm were identified in four patients, but were not included in the analyses. No previously known, but untreated UIA, were identified.

Of the 103 UIAs, $40(38.8 \%)$ were located in the anterior cerebral or anterior communicating arteries, 29 (28.2\%) in the middle cerebral artery, $22(21.4 \%)$ in the (intracranial) internal carotid artery and $12(11.7 \%)$ in the posterior cerebral, posterior communicating or basilar arteries. Of the 103 UIAs, 60 (58.3\%) were $\leq 3 \mathrm{~mm}, 24(23.3 \%)$ were $4-6 \mathrm{~mm}$ and 19 (18.4\%) were $\geq 7 \mathrm{~mm}$ in diameter (mean diameter $/ \mathrm{SD}=4.2 / 2.3 \mathrm{~mm}$ ). Patients with UIA were older than those without, more likely to be female and had a higher burden of hypertension $($ all $\mathrm{p}<0.05)$ (table 1). There were no differences in imaging modality in patients with and without UIA (table 1). In the 82 patients (86.3\%) with definite TIA/ischaemic stroke and UIA, 9 (11.0\%) were in the same territory as the presenting cerebrovascular event. In the 13 patients (13.7\%) with 'stroke mimics' and UIA, four had a discharge diagnosis of migraine, one with syncope, two with microvascular neuropathy, one with peripheral radiculopathy, three were unclear and two had functional neurological symptoms.

Female sex, current smoking and hypertension were independent predictors of UIA (table 2). There was a significant trend of increasing prevalence of UIA with the presence of these risk factors (online supplemental figure 2); $\mathrm{p}_{\text {trend }}<0.0001$ ). This was trend driven by female patients; if hypertensive and a smoker, the prevalence of UIA was $11.1 \%$ (figure 1). However, there was no difference in UIA size between male and female patients (mean/SD diameter 4.3/2.6 $\mathrm{mm}$ and $4.3 / 2.3 \mathrm{~mm}$, respectively).

Other baseline characteristics stratified by the presence of UIA and discharge diagnosis are shown in online supplemental table 2. The prevalence of UIA was higher in patients with a discharge diagnosis of definite minor stroke/TIA (5.1\%) compared with possible mimics (3.2\%). Prevalence of UIA was unrelated to age $\left(\mathrm{p}_{\text {trend }} 0.18\right.$; online supplemental figure 3$)$.

Two patients with UIA had a history of SAH from a different intracranial aneurysm. One patient had a family history of SAH (in a first-degree relative) and three patients had a possible family history of SAH (brain haemorrhage with unknown aetiology).

All scans with UIA were reviewed in a multidisciplinary meeting. Action was required in $44(46.3 \%)$ patients $(28 / 63.6 \%$ female): surveillance with interval scanning $(n=37)$ and
Table 1 Baseline population characteristics stratified by the presence of asymptomatic unruptured intracranial aneurysm

\begin{tabular}{|c|c|c|c|}
\hline \multirow[b]{2}{*}{ Characteristic } & \multicolumn{2}{|c|}{$\begin{array}{l}\text { Patients with intracranial vascular } \\
\text { imaging }(\mathrm{N}=2013)\end{array}$} & \multirow[b]{2}{*}{$P$ value } \\
\hline & No UIA $(\mathrm{N}=1918)^{*}$ & UIA (N=95) & \\
\hline Mean age (SD) & $67.2(15.2)$ & $70.4(12.9)$ & 0.044 \\
\hline Male sex (\%) & $979(51.0)$ & $30(31.6)$ & $<0.0001$ \\
\hline Caucasian (\%) & $1799(93.8)$ & $91(95.8)$ & 0.66 \\
\hline Hypertension (\%) & $975(50.8)$ & $60(63.2)$ & 0.019 \\
\hline Diabetes mellitus (\%) & $239(12.5)$ & $12(12.6)$ & 0.96 \\
\hline Hyperlipidemia (\%) & $608(31.7)$ & $27(28.4)$ & 0.50 \\
\hline Current smoker (\%) & $265(13.8)$ & $19(20.0)$ & 0.09 \\
\hline Atrial fibrillation (\%) & $261(13.6)$ & $9(9.5)$ & 0.25 \\
\hline Any vascular diseaset (\%) & $510(26.6)$ & $26(27.4)$ & 0.87 \\
\hline History of stroke or TIA (\%) & $263(13.7)$ & $16(16.8)$ & 0.36 \\
\hline PVD (\%) & $107(5.6)$ & $3(3.2)$ & 0.31 \\
\hline IHD (\%) & $224(11.7)$ & $8(8.4)$ & 0.33 \\
\hline \multicolumn{4}{|l|}{ Event type } \\
\hline TIA (\%) & $970(50.6)$ & $52(54.7)$ & 0.28 \\
\hline Minor stroke (\%) & $557(29.0)$ & $30(31.6)$ & \\
\hline Other diagnosis (\%) & $391(20.4)$ & $13(13.7)$ & \\
\hline \multicolumn{4}{|l|}{ Imaging modality } \\
\hline CTA $(\%)$ & 339 (17.7) & $16(16.8)$ & 0.51 \\
\hline MRA (\%) & $1579(82.3)$ & 79 (83.2) & \\
\hline
\end{tabular}

${ }^{*}$ Including 1 patient with previously treated ruptured intracranial aneurysm and 2 patients with non-saccular (fusiform) aneurysms.

TVascular disease $=$ prior ischaemic stroke/TIA, PVD or IHD.

CTA, computed tomography angiography; IHD, ischaemic heart disease; MRA, magnetic resonance angiography; PVD, peripheral vascular disease; TIA, transient ischaemic attack; UIA, unruptured intracranial aneurysm.

endovascular coiling $(n=7)$. There were seven treated UIAs in those who underwent endovascular coiling: three middle cerebral artery, two basilar artery, one posterior communicating artery and one anterior communicating artery UIA, of mean size $7.3 \mathrm{~mm}$ (range $5-11 \mathrm{~mm}$ ). Fifty patients $(52.6 \%)$ were discussed at MDT but no follow-up imaging was required, either because they were considered low risk $(n=40)$ or because the aneurysm or patient was considered too high risk for intervention $(n=10)$.

Of the 95 patients with UIA, 1 (1.1\%) had an SAH and 18 (18.9\%) died of other causes during mean follow-up of 4.5 years (SD 2.4). One (1.1\%) patient was lost to follow-up. Most identified UIAs $(88.3 \%)$ had a predicted 5 -year risk of rupture of less than $2 \%$ according to the PHASES risk score (table 3 ) and the overall average predicted risk was $0.9 \%$. The observed 5 -year risk of rupture in our cohort was $1.3 \%$ or 2.3 (95\% CI 0.3 to 16.6) per 1000 person-years.

\section{Systematic review and meta-analysis}

Nineteen studies comprising 12781 patients with ischaemic stroke/TIA were identified in the systematic review (online supplemental figure 1). These were studies of either patients undergoing emergency investigation for acute major ischaemic stroke $(n=12)$ or angiography for symptomatic carotid artery stenosis $(n=7)$ (online supplemental table 3$)$.

The mean age of patients in all studies was 62.9 years (three studies did not report mean age of participants) and $41.6 \%$ of participants were female (five studies did not report the proportion of females). In studies of patients under investigation for symptomatic carotid artery stenosis $(n=7)$, the mean UIA prevalence was $4.4 \%$ (95\% CI 3.9 to 4.9 ). In studies of patients under investigation for acute major ischaemic stroke $(n=12)$, the mean 
Table 2 Predictors of the presence of asymptomatic unruptured intracranial aneurysms

\begin{tabular}{|c|c|c|c|c|c|c|}
\hline & Unadjusted risk indicators & & Age-adjusted risk indicators & & Multivariable risk indicators* & \\
\hline & OR $(95 \% \mathrm{Cl})$ & $P$ value & $\mathrm{OR}(95 \% \mathrm{Cl})$ & $P$ value & OR $(95 \% \mathrm{Cl})$ & $P$ value \\
\hline Age (per 10 years) & $1.15(0.98$ to 1.36$)$ & 0.083 & - & & $1.13(0.95$ to 1.36$)$ & 0.18 \\
\hline Hypertension & 1.66 (1.08 to 2.54$)$ & 0.020 & 1.54 (0.98 to 2.40$)$ & 0.060 & 1.59 (1.01 to 2.50$)$ & 0.043 \\
\hline Current smoker & $1.56(0.93$ to 2.62$)$ & 0.093 & 1.20 (1.03 to 1.39$)$ & 0.021 & 2.07 (1.19 to 3.60$)$ & 0.010 \\
\hline Female sex & $2.26(1.45$ to 3.51$)$ & $<0.0001$ & 2.21 (1.42 to 3.44$)$ & $<0.0001$ & 2.34 (1.50 to 3.66$)$ & $<0.0001$ \\
\hline
\end{tabular}

*The multivariable analyses includes each of the risk factors listed.

UIA prevalence was $5.9 \%$ (95\% CI 5.3 to 6.5 ). The mean pooled UIA prevalence in all studies (including the OXVASC cohort patients with TIA/minor stroke) was 5.1\% (95\% CI 4.8 to 5.5, figure 2$)$, but there was significant heterogeneity $(\mathrm{p}<0.00001)$.

Of the studies that reported UIA management $(n=7), 80$ patients with UIA (19.2\%) underwent intervention. Only six studies provided sufficient information for the risk of subsequent SAH and length of participant follow-up (table 4); of these, there were four SAH in 217 patients over a mean follow-up of 2.5 years. The overall incidence of SAH (including the OXVASC data) was 4.6 (95\% CI 1.9 to 11.0 ) per 1000 person-years.

\section{DISCUSSION}

In this first population-based study of patients with suspected TIA and minor stroke, we found an overall prevalence of asymptomatic UIA of $4.7 \%$, increasing to $11.1 \%$ in hypertensive females who smoked. Although nearly half the patients found to have an UIA required additional investigations or procedures, there was only one SAH during follow-up. This is consistent with the overall predicted 5 -year aneurysm rupture risk of $0.9 \%$ determined by the mean PHASES score.

UIA are formed by a combination of degenerative factors such as haemodynamic stress, a genetic predisposition and vascular risk factors, including hypertension, lipid accumulation and smoking. ${ }^{16}$ The largest meta-analysis to date of the prevalence of UIA included 95000 individuals and reported an overall rate of $3.2 \%$, higher in those with a background of atherosclerosis. ${ }^{1}$ Accordingly, our systematic review of UIA prevalence in patients with cerebrovascular disease demonstrated a UIA prevalence of $5.1 \%$, although with significant heterogeneity. This is probably due to an excess of vascular risk factors, as demonstrated by patients with UIA in our cohort having similar baseline characteristics, irrespective of the discharge diagnosis, apart from a

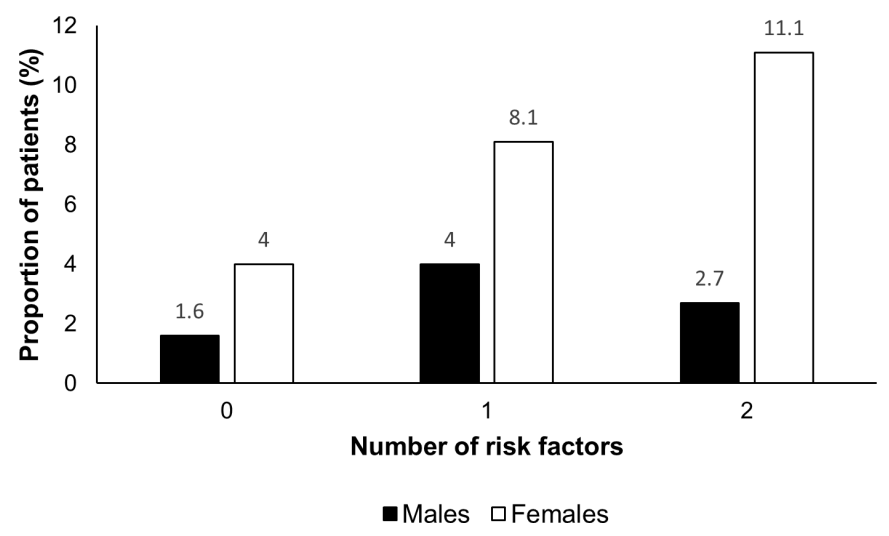

Figure 1 Prevalence of patients with unruptured intracranial aneurysms categorised by the number of risk factors (female sex, hypertension and current smoker) and sex. higher burden of hypertension in the non-stroke group (online supplemental table 2).

The significant heterogeneity in prevalence between previous studies is likely explained by the differing selection criteria of the acute ischaemic stroke studies. These studies variably selected consecutive patients with acute ischaemic stroke $(n=6)$, or based on eligibility for thrombolysis $(n=4)$ or thrombectomy $(n=2)$. All studies were hospital based and ours is the only study to report prevalence and prognosis in patients with TIA and minor ischaemic stroke.

Risk factors for aneurysm growth and rupture are similar to those for UIA formation, with the addition of size and site. ${ }^{16}$ Most aneurysms identified in our cohort were estimated to be low risk which was consistent with the low rate of aneurysm rupture in our cohort. In addition, there may be protective antiinflammatory effects of aspirin further reducing the risk of UIA rupture in these patients ${ }^{5-7}$; a clinical trial is ongoing (unique identifier: NCT02846259). ${ }^{17}$ There is a significant lack of prognostic data currently available-only six studies identified in the systematic review provided information regarding SAH risk and only two of these reported any outcome events (table 4). The power of our cohort was similar to the largest of these studies and demonstrated a similar risk of SAH: 2.3 and 2.2 per 1000 person-years, respectively.

The clinical implications of our findings are that clinicians should be aware of the higher prevalence of UIA in patients with cerebrovascular disease and emphasise risk factor control. Moreover, neuroradiologists should be attentive to the possible presence of UIA in patients with TIA/stroke, particularly females with other risk factors. Although there is insufficient evidence

Table 3 Unruptured intracranial aneurysms in the OXVASC cohort stratified by 5 -year risk of rupture calculated with PHASES score (taken from Greving et $\mathrm{al}^{14}$ )

\begin{tabular}{ccc}
\hline PHASES score & $\begin{array}{l}\text { Predicted 5-year risk of } \\
\text { aneurysm rupture }(95 \% \mathrm{Cl})\end{array}$ & $\begin{array}{l}\text { Number of UIAs in OXVASC } \\
\text { cohort, } \mathrm{n}=103 \text { (\%) }\end{array}$ \\
\hline$\leq 2$ & $0.4 \%(0.1$ to 1.5$)$ & $18(17.5)$ \\
\hline 3 & $0.7 \%(0.2$ to 1.5$)$ & $9(8.7)$ \\
\hline 4 & $0.9 \%(0.3$ to 2.0$)$ & $26(25.2)$ \\
\hline 5 & $1.3 \%(0.8$ to 2.4$)$ & $12(11.7)$ \\
\hline 6 & $1.7 \%(1.1$ to 2.7$)$ & $26(25.2)$ \\
\hline 7 & $2.4 \%(1.6$ to 3.3$)$ & $4(3.9)$ \\
\hline 8 & $3.2 \%(2.3$ to 4.4$)$ & $2(1.9)$ \\
\hline 10 & $4.3 \%(2.9$ to 6.1$)$ & $4(3.9)$ \\
\hline 11 & $5.3 \%(3.5$ to 8.0$)$ & $2(1.9)$ \\
$\geq 12$ & $7.2 \%(5.0$ to 10.2$)$ & $0(0.0)$ \\
\hline OXVASC, $0 x$. & $17.8(15.2$ to 20.7$)$ & $0(0.0)$ \\
\hline
\end{tabular}

OXVASC, Oxford Vascular Study; PHASES, population, hypertension, age, size of aneurysm, previous subarachnoid haemorrhage (from a different aneurysm), site of aneurysm; UIA, unruptured intracranial aneurysm. 


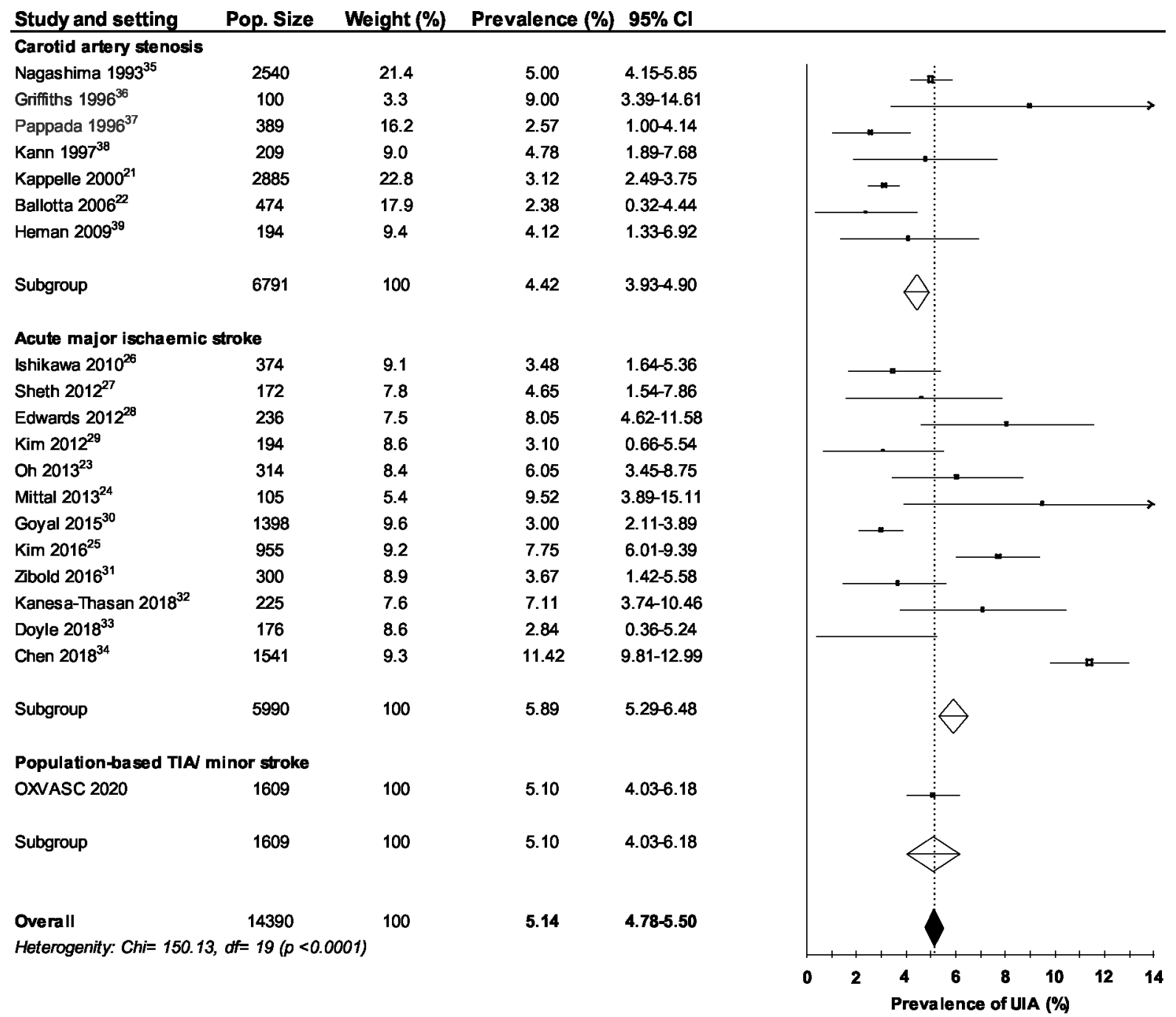

Figure 2 Forest plot depicting the prevalence of unruptured intracranial aneurysms in patients with ischaemic stroke or transient ischaemic attack (TIA), categorised by study selection criteria. ${ }^{23-41}$

to suggest routine screening, the prevalence of UIA in female patients with minor stroke/TIA with other risk factors is higher than that of people in the general population with two or more first-degree relatives with SAH or UIA who are advised to undergo routine screening. ${ }^{18}$ Patients with TIA/stroke can be counselled that the overall risk of rupture is low during the next 5 years in the context of management according to American
Heart Association/American Stroke Association guidelines for incidental aneurysms more generally, and might be lowered further by medication compliance and smoking cessation. A standard approach to UIA management appears to be applicable to this cohort.

The strengths of our study include its population-based nature with high rates of ascertainment and near complete investigation

\begin{tabular}{|c|c|c|c|c|c|}
\hline Study/setting & $\begin{array}{l}\text { Recruitment } \\
\text { period }\end{array}$ & $\begin{array}{l}\text { Number of patients } \\
\text { with UIA (\%) }\end{array}$ & $\begin{array}{l}\text { Person-years } \\
\text { follow-up }\end{array}$ & $\begin{array}{l}\text { Number of } \\
\text { subsequent SAHs }\end{array}$ & $\begin{array}{l}\text { Risk of SAH (per } 1000 \\
\text { person-years) }\end{array}$ \\
\hline Kappelle $\mathrm{e}^{23}$ & 1988-1991 & $90(3.1)$ & 450.0 & 1 & 2.2 \\
\hline Ballotta $^{24}$ & 1992-1999 & $11(2.3)$ & 55.0 & 0 & 0 \\
\hline \multicolumn{6}{|c|}{ Acute major ischaemic stroke } \\
\hline $\mathrm{Kim}^{27}$ & 2011-2014 & $74(7.7)$ & 111.0 & 3 & 27.1 \\
\hline \multicolumn{6}{|c|}{ Population-based TIA/minor stroke } \\
\hline OXVASC 2020 & $2011-2020$ & $95(4.7)$ & 427.5 & 1 & 2.3 \\
\hline Total & - & $312(5.3)$ & 1092.3 & 5 & 4.6 \\
\hline
\end{tabular}

SAH, subarachnoid haemorrhage; UIA, unruptured intracranial aneurysm. 
and follow-up. Patients were reviewed by a single senior neurologist and imaging was reported by a single neuroradiologist to maximise diagnostic consistency. More than $80 \%$ of eligible patients underwent intracranial vascular imaging, mostly MRA, which has a high sensitivity in detecting UIA and is comparable with that of CTA. ${ }^{19}$ Subsequent management of UIA reflected American Heart Association/American Stroke Association guidelines.

However, our study does have some limitations. First, some patients did not receive intracranial vascular imaging, but this was due to unavoidable issues (such as severe dementia, patient refusal or contraindication to contrast media) or patients who presented with unambiguous mimics (often with few vascular risk factors), and so our findings can still be applied to a typical neurovascular clinic setting. Second, although more than $80 \%$ of patients in our study received our first preference of MRI/ MRA brain, CTA had to be used when MRA was contraindicated. However, there was no difference in the proportion of patients receiving either modality in the UIA and no UIA groups. Third, the UIA cohort was not powered to provide a precise estimate of prognosis. However, ours is the largest study so far on the prevalence and prognosis of incidental UIA in patients with cerebrovascular disease, although future studies with longer follow-up will be required to confirm the natural history UIA in this group. Fourth, although our results suggest that noninvasive angiography should be performed to screen for UIA in higher-risk patients, we are not able to conclude that this affects the risk of subsequent SAH and therefore improves patient outcome. Moreover, the psychological burden on patients who are found to have an incidental UIA, which may be small and will not require further investigation, should not be understated. ${ }^{20}$ Fifth, our study represents one centre's management approach, and although it is consistent with international guidelines, monitoring and intervention might be more intensive in other healthcare systems. However, the prognosis of our patients was good despite less intensive monitoring than in some centres, suggesting that our findings are likely to be generalisable. Sixth, although there are some small studies suggesting a role of symptomatic arterial embolisation from UIA, ${ }^{21} 22$ we did not prospectively determine their potential role in the aetiology of the presenting ischaemic event in our study. However, as only $11 \%$ of UIAs were in the same arterial territory as the presenting event, this is likely to be a rare phenomenon. Seventh, we have only included patients with minor or transient events in this study and our findings are not translatable to patients with major ischaemic stroke. However, our cohort was selected based on the lack of data in these patients, the high rates of investigation enabling an accurate estimation of UIA prevalence and those in whom the identification of an UIA would have the greatest clinical implication.

In conclusion, the overall prevalence of UIA in a TIA and minor stroke cohort is probably higher than in the general population. However, in the context of intensively managed vascular risk factors and a standard policy for surveillance and intervention, the risk of rupture is low. These findings should be considered when counselling patients and in service design.

\footnotetext{
Acknowledgements We thank all primary care practices and physicians who collaborated with the Oxford Vascular Study. The Oxford Vascular Study has been funded by the Association of British Neurologists, Wellcome Trust, Wolfson Foundation, UK Stroke Association, British Heart Foundation, Dunhill Medical Trust, National Institute of Health Research (NIHR), Medical Research Council and the NIHR Oxford Biomedical Research Centre. We also acknowledge the use of the facilities of the Acute Vascular Imaging Centre, Oxford.
}

Contributors RH was responsible for acquisition of data, analysis and interpretation and drafting of the manuscript. WK was responsible for imaging analysis. PMR was responsible for study conception, design, supervision, funding, analysis and interpretation of data and drafting of the manuscript. Other authors were responsible for acquisition of data and for revision of the manuscript.

Funding Wellcome Trust, Wolfson Foundation, British Heart Foundation, National Institute for Health Research, National Institute for Health Research Oxford Biomedical Research Centre and the Association of British Neurologists.

Disclaimer No funding body had any role in the design, data collection, analysis, interpretation or writing of this study nor were involved in the decision to submit for publication.

Competing interests None declared.

Patient consent for publication Not required.

Ethics approval OXVASC was approved by the Oxfordshire research ethics committee (OREC A: 05/Q1604/70).

Provenance and peer review Not commissioned; externally peer reviewed.

Data availability statement Data are available on reasonable request. All data relevant to the study are included in the article or uploaded as online supplemental information. Requests for access to the data reported in this paper will be considered by the corresponding author.

Supplemental material This content has been supplied by the author(s). It has not been vetted by BMJ Publishing Group Limited (BMJ) and may not have been peer-reviewed. Any opinions or recommendations discussed are solely those of the author(s) and are not endorsed by BMJ. BMJ disclaims all liability and responsibility arising from any reliance placed on the content. Where the content includes any translated material, BMJ does not warrant the accuracy and reliability of the translations (including but not limited to local regulations, clinical guidelines, terminology, drug names and drug dosages), and is not responsible for any error and/or omissions arising from translation and adaptation or otherwise.

Open access This is an open access article distributed in accordance with the Creative Commons Attribution 4.0 Unported (CC BY 4.0) license, which permits others to copy, redistribute, remix, transform and build upon this work for any purpose, provided the original work is properly cited, a link to the licence is given, and indication of whether changes were made. See: https://creativecommons.org/ licenses/by/4.01.

\section{ORCID iDs}

Robert Hurford http://orcid.org/0000-0002-4226-7681

Wilhelm Kuker http://orcid.org/0000-0002-1739-6115

\section{REFERENCES}

1 Vlak MH, Algra A, Brandenburg R, et al. Prevalence of unruptured intracranial aneurysms, with emphasis on sex, age, comorbidity, country, and time period: a systematic review and meta-analysis. Lancet Neurol 2011;10:626-36.

2 Vernooij MW, Ikram MA, Tanghe HL, et al. Incidental findings on brain MRI in the general population. N Engl J Med 2007;357:1821-8.

3 Smedley A, Yusupov N, Almousa A, et al. Management of incidental aneurysms: comparison of single centre multi-disciplinary team decision making with the unruptured incidental aneurysm treatment score. Br J Neurosurg 2018;32:536-40.

4 Griessenauer CJ, Farrell S, Sarkar A, et al. Genetic susceptibility to cerebrovascular disease: a systematic review. J Cereb Blood Flow Metab 2018;38:1853-71.

5 Hasan DM, Chalouhi N, Jabbour P, et al. Evidence that acetylsalicylic acid attenuates inflammation in the walls of human cerebral aneurysms: preliminary results. J Am Heart Assoc 2013:2:e000019.

6 Hasan DM, Mahaney KB, Brown RD, et al. Aspirin as a promising agent for decreasing incidence of cerebral aneurysm rupture. Stroke 2011;42:3156-62.

7 Hudson JS, Marincovich AJ, Roa JA, et al. Aspirin and intracranial aneurysms. Stroke United States, 2019: 2591-6.

8 Bamford J, Sandercock P, Dennis M, et al. A prospective study of acute cerebrovascular disease in the community: the Oxfordshire Community Stroke Project-1981-86. 2 Incidence, case fatality rates and overall outcome at one year of cerebral infarction, primary intracerebral and subarachnoid haemorrhage. J Neurol Neurosurg Psychiatry 1990:53:16-22

9 Rothwell PM, Coull AJ, Silver LE, et al. Population-based study of event-rate, incidence, case fatality, and mortality for all acute vascular events in all arterial territories (Oxford vascular study). Lancet 2005;366:1773-83.

10 Coull AJ, Silver LE, Bull LM, et al. Direct assessment of completeness of ascertainment in a stroke incidence study. Stroke 2004;35:2041-5.

11 Adams HP, Bendixen BH, Kappelle LJ, et al. Classification of subtype of acute ischemic stroke. definitions for use in a multicenter clinical trial. TOAST. Trial of Org 10172 in Acute Stroke Treatment. Stroke 1993;24:35-41.

12 WHO. Cerebrovascular diseases - prevention, treatment and rehabilitation. Technical Report Series no 469. Geneva: WHO, 1971 
13 Lau K-K, Li L, Lovelock CE, et al. Clinical correlates, ethnic differences, and prognostic implications of perivascular spaces in transient ischemic attack and ischemic stroke. Stroke 2017;48:1470-7.

14 Greving JP, Wermer MJH, Brown RD, et al. Development of the PHASES score for prediction of risk of rupture of intracranial aneurysms: a pooled analysis of six prospective cohort studies. Lancet Neurol 2014;13:59-66.

15 Thompson BG, Brown RD, Amin-Hanjani S, et al. Guidelines for the management of patients with unruptured intracranial aneurysms: a guideline for healthcare professionals from the American Heart Association/American Stroke Association. Stroke 2015;46:2368-400.

16 Etminan N, Rinkel GJ. Unruptured intracranial aneurysms: development, rupture and preventive management. Nat Rev Neurol 2016;12:699-713.

17 Etminan N. Acetylsalicylic acid plus intensive blood pressure treatment in patients with unruptured intracranial aneurysms (PROTECT-U) [online], 2017. Available: https:// www.clinicaltrials.gov/ct2/show/NCT03063541?term =NCT03063541\&draw=2\& rank=1 [Accessed 1 Jun 2020].

18 Rinkel GJE. Intracranial aneurysm screening: indications and advice for practice. Lancet Neurol 2005;4:122-8.

19 Sailer AMH, Wagemans BAJM, Nelemans PJ, et al. Diagnosing intracranial aneurysms with MR angiography: systematic review and meta-analysis. Stroke 2014;45:119-26.

20 van der Schaaf IC, Brilstra EH, Rinkel GJE, et al. Quality of life, anxiety, and depression in patients with an untreated intracranial aneurysm or arteriovenous malformation. Stroke 2002;33:440-3.

21 McLaughlin N, Bojanowski MW. Unruptured cerebral aneurysms presenting with ischemic events. Can J Neurol Sci 2008;35:588-92.

22 Friedman JA, Piepgras DG, Pichelmann MA, et al. Small cerebral aneurysms presenting with symptoms other than rupture. Neurology 2001;57:1212-6.

23 Kappelle LJ, Eliasziw M, Fox AJ, et al. Small, unruptured intracranial aneurysms and management of symptomatic carotid artery stenosis. North American Symptomatic Carotid Endarterectomy Trial Group. Neurology 2000;55:307-9.

24 Ballotta E, Da Giau G, Manara R, et al. Extracranial severe carotid stenosis and incidental intracranial aneurysms. Ann Vasc Surg 2006;20:5-8.

25 Oh Y-S, Shon Y-M, Kim BS, et al. Long-term follow-up of incidental intracranial aneurysms in patients with acute ischemic stroke. J Stroke Cerebrovasc Dis 2013:22:329-33.

26 Mittal MK, Seet RCS, Zhang Y, et al. Safety of intravenous thrombolysis in acute ischemic stroke patients with saccular intracranial aneurysms. J Stroke Cerebrovasc Dis 2013;22:639-43.
$27 \mathrm{Kim} \mathrm{JH}$, Suh SH, Chung J, et al. Prevalence and characteristics of unruptured cerebral aneurysms in ischemic stroke patients. J Stroke 2016;18:321-7.

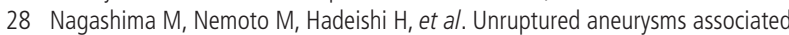
with ischaemic cerebrovascular diseases. surgical indication. Acta Neurochir 1993; 124:71-8.

29 Griffiths PD, Worthy S, Gholkar A. Incidental intracranial vascular pathology in patients investigated for carotid stenosis. Neuroradiology 1996;38:25-30.

30 Pappadà G, Fiori L, Marina R, et al. Management of symptomatic carotid stenoses with coincidental intracranial aneurysms. Acta Neurochir 1996;138:1386-90.

31 Kann BR, Matsumoto T, Kerstein MD. Safety of carotid endarterectomy associated with small intracranial aneurysms. South Med J 1997;90:1213-6.

32 Héman LM, Jongen LM, van der Worp HB, et al. Incidental intracranial aneurysms in patients with internal carotid artery stenosis: a CT angiography study and a metaanalysis. Stroke 2009;40:1341-6.

33 Ishikawa Y, Hirayama T, Nakamura Y, et al. Incidental cerebral aneurysms in acute stroke patients: comparison of asymptomatic healthy controls. J Neurol Sci 2010;298:42-5.

34 Sheth KN, Shah N, Morovati T, et al. Intravenous rt-PA is not associated with increased risk of hemorrhage in patients with intracranial aneurysms. Neurocrit Care 2012;17:199-203.

35 Edwards NJ, Kamel H, Josephson SA. The safety of intravenous thrombolysis for ischemic stroke in patients with pre-existing cerebral aneurysms: a case series and review of the literature. Stroke 2012;43:412-6.

36 Kim J-T, Park M-S, Yoon W, et al. Detection and significance of incidental unruptured cerebral aneurysms in patients undergoing intravenous thrombolysis for acute ischemic stroke. J Neuroimaging 2012;22:197-200.

37 Goyal N, Tsivgoulis G, Zand R, et al. Systemic thrombolysis in acute ischemic stroke patients with unruptured intracranial aneurysms. Neurology 2015;85:1452-8.

38 Zibold F, Kleine JF, Zimmer C, et al. Aneurysms in the target vessels of stroke patients subjected to mechanical thrombectomy: prevalence and impact on treatment. J Neurointerv Surg 2016;8:1016-20.

39 Kanesa-Thasan R, Cox M, Patel M, et al. Actionable vascular and other incidental findings on CTA in patients undergoing acute stroke intervention. Neuroradiol J 2018;31:572-7.

40 Doyle SJ, George BP, Holloway RG, et al. Incidental findings in radiographic imaging for inpatients with acute ischemic stroke. J Stroke Cerebrovasc Dis 2018;27:3131-6.

41 Chen ML, Gupta A, Chatterjee A, et al. Association between unruptured intracranial aneurysms and downstream stroke. Stroke 2018;49:2029-33. 